\section{Temporal lobe atypical teratoid/ rhabdoid tumor in a 24-year old adult female}

Dear Editor,

The name atypical teratoid/rhabdoid tumor (AT/RT), a histological variant of Wilm's tumor exemplifies the tumor's disparate mixtures of rhabdoid, primitive neuroepithelial, mesenchymal and epithelial components. AT/RT is much more frequently seen in infants and young children and is rare in adults. Prognosis of AT/RT is poor with most of the patients dying shortly after the diagnosis. ${ }^{[1]}$ The literature suggests that the use of more aggressive therapies (including surgery, chemotherapy with or without stem-cell support, intrathecal chemotherapy, and early radiotherapy), may lead to some prolongation of the natural history of this tumor. ${ }^{[2,3]}$

A 24-year-old female was admitted in the neurosurgery ward of Institute of Post Graduate Medical Education and Research and S.S.K.M Hospital, Kolkata with complaints of repeated headache for 2 months, few episodes of vomiting and unable to open her right eye for 1 month. On general physical examination patient was conscious, afebrile, with stable vital signs and good hydration. There was mild pallor, with no cyanosis, edema, and icterus. On central nervous system examination, there was right sided third cranial nerve palsy. No other neurological deficit was found. Other systemic examinations were within normal limits. Magnetic resonance imaging brain showed a mass lesion in the medial aspect of right temporal lobe and in suprasellar region [Figure 1]. After 10 days, right temporal craniotomy with gross excision of the tumor was done under general anesthesia. Operative specimen was sent for histopathological examination and immuno-histochemistry. Section showed cerebral tissue and a tumor composed of rhabdoid cells in abundant myxoid matrix, cells were round to spindle with a moderate amount of eosinophilic granular cytoplasm with hyper chromatic nuclei [Figure 2] suggestive of AT/RT (World Health Organization Grade four). Immuno-histochemistry depicted that tumor cells were positive for vimentin, epithelial membrane antigen, S-100, and glial fibrillary acidic protein (GFAP) and immune-negativity for CD-31; CD-34 and cytokeratin with loss of expression of nuclear INI1 protein. The Mib-1 labeling index was $12-15 \%$. After 2 weeks of surgery, a computed tomography scan brain was done and showed ill-defined residual mass in right medial temporal lobe and post-operative changes in right temporal region [Figure 3]. However, after surgery general condition of the patient did not improve satisfactorily and the patient was referred for adjuvant therapy. A post-operative chest X-ray, ultra-sonography abdomen and routine blood parameters were all within normal limits. The patient underwent adjuvant craniospinal irradiation (CSI) to $36 \mathrm{~Gy}$, followed by boost treatment at tumor bed up to 54 Gy using conventional fractionation of $1.8 \mathrm{~Gy} /$ day. Then she received 6 cycles of adjuvant chemotherapy with Ifosfamide $\left(1800 \mathrm{mg} / \mathrm{m}^{2} \times \operatorname{Day}_{1-4}\right)$, Etoposide $\left(100 \mathrm{mg} / \mathrm{m}^{2} \times \operatorname{Day}_{1-4}\right)$ and Carboplatin (Area Under the Curve AUC 5, Day ${ }_{1-2}$ ) at an interval of 21 days. She remains clinically and radio graphically stable 11 months after the initial diagnosis.

Rhabdoid tumor was originally described as a variant of Wilms' tumor with rhabdomyosarcomatous features. Subsequently, the features differentiating AT/RT from Wilms' tumor were clarified. ${ }^{[2,4,5]}$ The most frequent anatomic location for non-renal rhabdoid tumor is the CNS. Report from the AT/RT workshop in 2002 noted that half of all AT/RTs was in the posterior fossa, although, the tumor has been noted throughout the nervous system and in extramedullary sites. ${ }^{[3]}$ The association of renal and CNS AT/RT was consistent with cytogenetic studies that demonstrated abnormalities of 22q11.2 in tumors from both sites. ${ }^{[2,6]}$ The mean age of the patients is 2.9 years with a male predominance. ${ }^{[2,6]}$ There are very few adult cases reported in the literature to date, most of them are male. In adult females, the tumor is most commonly located at sites other than the temporal lobe. ${ }^{[3]}$ In our case, patient was a 24-year-old female with temporal lobe AT/RT.

Molecular genetic studies have led to the identification of a rhabdoid suppressor gene (INI1/hSNF5) at 22q11.2. Somatic mutations in this gene predispose children to the development of AT/RT. There are reports of adults with AT/RT in the literature, although molecular genetic studies have not yet confirmed the involvement of INI1 in an adult. ${ }^{[2]}$ However, molecular genetic analysis of the INI1 gene may be useful in confirming the diagnosis of AT/RT. ${ }^{[3]}$ The histopathology spectrum of AT/RT is broad, ranging from predominantly small cells with primitive morphology to tumors with large rhabdoid cells. In addition, some AT/RTs may have mesenchymal and epithelial components. The rhabdoid cells have prominent nucleoli and conspicuous, spherical, cytoplasmic inclusions. The immunophenotypic

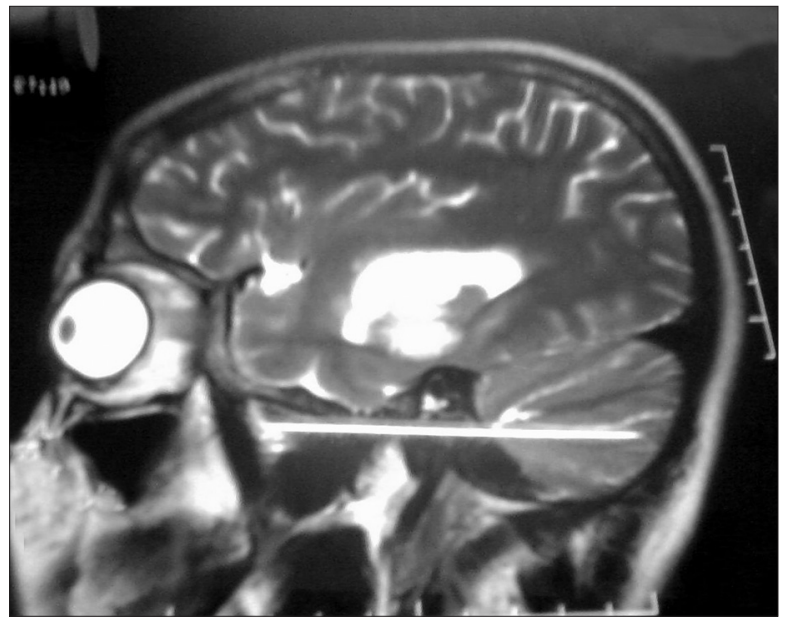

Figure 1: Pre-operative contras enhanced magnetic resonance imaging (sagittal section) showing a mass lesion in medial aspect of right temporal lobe and in suprasellar region 


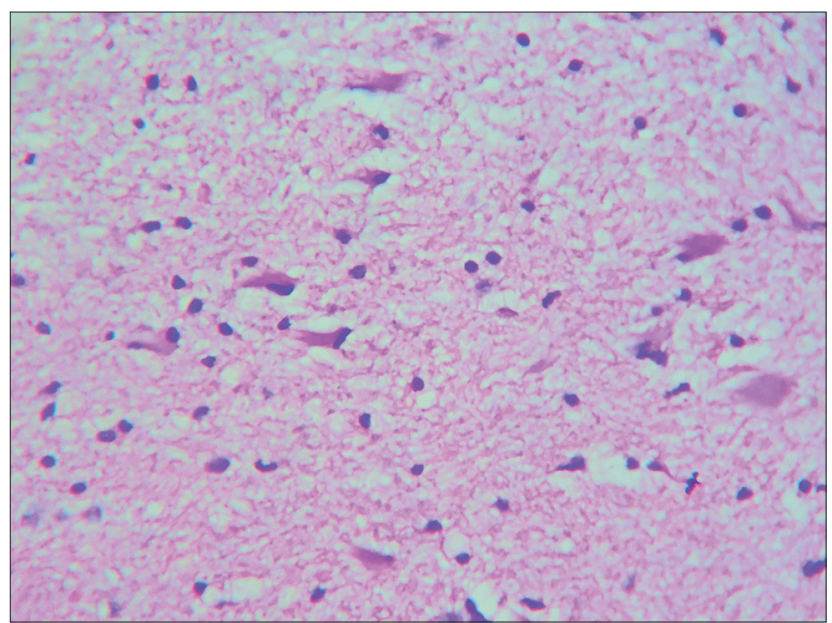

Figure 2: Histopathology slide showing plenty of rhabdoid cells in abundant myxoid matrix

profile of AT/RT is broad, showing variable reactivity with epithelial membrane antigen, GFAP, cytokeratins, and less frequently with actin, neurofilaments, and chromogranin..$^{[3,7]}$

Since, its histological appearance can be confused with other tumors, especially in adults; separating AT/RT from other neoplasms may be difficult. In many instances, a reliable diagnosis is not possible without demonstrating the lack of nuclear INI1 protein expression by immunohistochemical methods. ${ }^{[8]}$ In our case, there was loss of nuclear expression of INI1 protein, confirmed the diagnosis of atypical AT/RT.

Treatment is highly variable, but most children receive the multi-modality therapy of surgery, radiation and chemotherapy. The degree of surgical resection appears to be one of the most important factors in predicting prognosis. Hilden et al. showed event free survival (EFS) of 20 patients who had a gross total resection was 14 months (range: 1.5-72 months) compared to the EFS of 9.25 months (range: 1-96 months) for the subset with a partial resection. They recommended that an aggressive surgical approach be undertaken, including, second look surgery when possible, to achieve gross total resection. ${ }^{[2]}$

Typically, children are treated with CSI which is often followed by systemic chemotherapy; commonly utilize vincristine with an alkylating and a platinum based regimens. Treatment paradigms for adult patients have been extracted from the pediatric literature. Much of the data regarding the treatment has focused on chemotherapy since the majority of patients diagnosed with AT/RT are under 2 to 3 years old when radiotherapy is avoided if possible. Chemotherapy regimens with ifosfamide, etoposide and carboplatin and temozolomide have been used in adults. ${ }^{[2,3]}$ Those patients over the age of three are routinely given radiotherapy, often in the form of CSI as leptomeningeal disease is often present at diagnosis and is common at recurrence. Patients are given varying doses between $40 \mathrm{~Gy}$ and $60 \mathrm{~Gy}$, and stereotactic radiosurgery has been used for recurrent disease when resection is

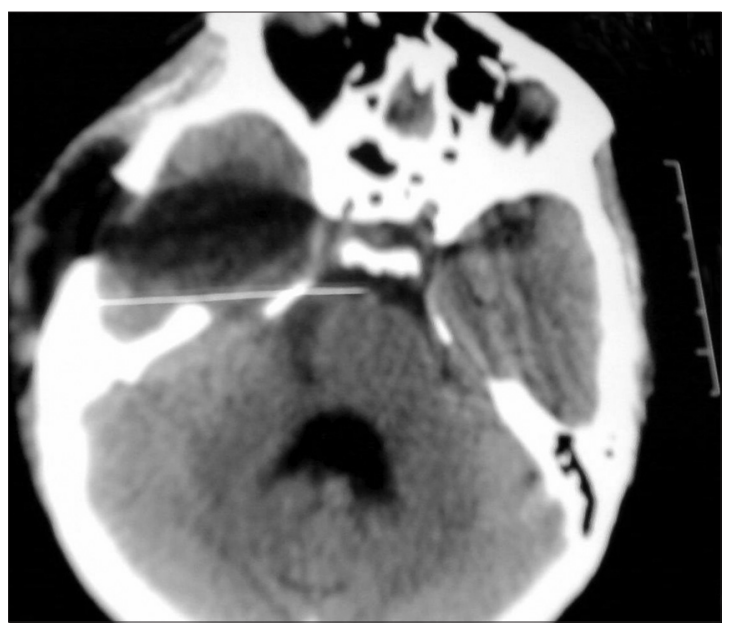

Figure 3: Post-operative computed tomography scan (plain) showing ill defined residual mass in right medial temporal lobe and post-operative changes in right temporal region

not feasible. ${ }^{[3,9]}$ Chen et al. recommended initiating radiotherapy immediately postoperatively and before chemotherapy in patients over 3 years of age. They showed longest survival among those groups underwent gross tumor removal, and completed both craniospinal, and focal boost irradiation. ${ }^{[10]}$

AT/RT is predominantly a childhood tumor and has only been rarely reported in adults; therefore, treatment regimens are often extrapolated from the pediatric experience. Aggressive resection followed by multimodality treatment appears to yield more long-term survivors. Although, the optimal chemotherapy regimen has not been defined for adults with AT/RT, several regimens have been used with evidence of activity and the use of radiation does not convey the same devastating developmental arrest of disease progression. Further advances in treatment will likely require more laboratory studies generating novel treatment regimens and a standardized treatment protocol for adult AT/RT.

\section{Somnath Roy, Chandrani Mallik, Sumana Maiti, Tamojit Chaudhuri'}

Departments of Radiotherapy, Institute of Post Graduate Medical Education and Research and SSKM Hospital, Kolkata, West Bengal, 'SGPGIMS, Lucknow, Utter Pradesh, India Correspondence to: Dr. Somnath Roy, E-mail: drsomnath1980@gmail.com

\section{References}

1. Sidhu M, Sakhuja P, Malhotra V, Gondal R. A typical teratoid/ rhabdoid tumour of brain. J K Sci 2003;5:81-3.

2. Hilden JM, Meerbaum S, Burger P, Finlay J, Janss A, Scheithauer BW, et al. Central nervous system atypical teratoid/rhabdoid tumor: Results of therapy in children enrolled in a registry. J Clin Oncol 2004;22:2877-84.

3. Shonka NA, Armstrong TS, Prabhu SS, Childress A, Choi S, Langford LA, et al. Atypical teratoid/rhabdoid tumors in adults: A case report and treatment-focused review. J Clin Med Res 2011;3:85-92.

4. Haas JE, Palmer NF, Weinberg AG, Beckwith JB. Ultrastructure of malignant rhabdoid tumor of the kidney. A distinctive renal tumor of children. Hum Pathol 1981;12:646-57. 
5. Weeks DA, Beckwith JB, Mierau GW, Luckey DW. Rhabdoid tumor of kidney. A report of 111 cases from the National Wilms tumor study pathology center. Am J Surg Pathol 1989;13:439-58.

6. Bilginer B, Bozkurt G, Akalan N, Soylemezoglu F, Zorlu F, Buyukpamukcu M, et al. Atypical teratoid/rhabdoid tumors. Turk Neurosurg 2006; 16:77.

7. Parwani AV, Stelow EB, Pambuccian SE, Burger PC, Ali SZ. Atypical teratoid/rhabdoid tumor of the brain: Cytopathologic characteristics and differential diagnosis. Cancer 2005; 105:65-70.

8. Makuria AT, Rushing EJ, McGrail KM, Hartmann DP, Azumi N, Ozdemirli M. Atypical teratoid rhabdoid tumor (AT/RT) in adults: Review of four cases. J Neurooncol 2008;88:321-30.

9. Olson TA, Bayar E, Kosnik E, Hamoudi AB, Klopfenstein KJ, Pieters RS, et al. Successful treatment of disseminated central nervous system malignant rhabdoid tumor. J Pediatr Hematol Oncol
1995; 17:71-5

10. Chen YW, Wong TT, Ho DM, Huang PI, Chang KP, Shiau CY, et al. Impact of radiotherapy for pediatric CNS atypical teratoid/rhabdoid tumor (single institute experience). Int J Radiat Oncol Biol Phys 2006;64:1038-43.

\begin{tabular}{|l|l|}
\hline \multicolumn{2}{|c|}{ Access this article online } \\
\hline Quick Response Code: & Website: \\
\hline
\end{tabular}

